\title{
Towards Resolving the Presynaptic NMDA Receptor Debate
}

Guy Bouvier ${ }^{1}$, Rylan S. Larsen², Antonio Rodríguez-Moreno ${ }^{3}$, Ole Paulsen ${ }^{4}$, and P. Jesper Sjöström ${ }^{5 *}$

1) University of California, San Francisco, Physiology Department, CA, USA

2) Allen Institute for Brain Science, Seattle, WA 98103, USA

3) Universidad Pablo de Olavide, Seville, Spain

4) University of Cambridge, Cambridge, United Kingdom

5) Centre for Research in Neuroscience, The BRAIN Program, Department of Neurology and Neurosurgery, The Research Institute of the McGill University Health Centre, Montreal General Hospital, Montréal, Québec, H3G 1A4, Canada

*) Correspondence: jesper.sjostrom@mcgill.ca

Running title: Presynaptic NMDA Receptors

Words: 2950 (excluding references and cover page)

Pages: $\quad 20$

Figures: $\quad 3$

\section{Address for editorial correspondence:}

Prof P J Sjöström

Centre for Research in Neuroscience

Brain Repair and Integrative Neuroscience Programme

Department of Neurology and Neurosurgery

The Research Institute of the McGill University Health Centre

Montreal General Hospital

1650 Cedar Ave, room L7-225

Montreal Quebec H3G 1A4, Canada

Office: +1-514-934-1934, ext. 44561

Mob: +1-438-826-1971

FAX: +1-514-934-8216 


\section{Abstract}

In the classical view, postsynaptic NMDA receptors (NMDARs) trigger Hebbian plasticity via $\mathrm{Ca}^{2+}$ influx. However, unconventional presynaptic NMDARs (preNMDARs) which regulate both long-term and short-term plasticity at several synapse types have also been found. A lack of sufficiently specific experimental manipulations and a poor understanding of how preNMDARs signal have contributed to long-standing controversy surrounding these receptors. Although several prior studies linked preNMDARs to neocortical timing-dependent long-term depression (tLTD), a recent study argues that the NMDARs are actually postsynaptic and signal metabotropically, i.e. without $\mathrm{Ca}^{2+}$. Other recent work indicates that, whereas ionotropic preNMDARs signaling controls evoked release, spontaneous release is regulated by metabotropic NMDAR signaling. We argue that elucidating unconventional NMDAR signaling modes — both presynaptically and metabotropically — is key to resolving the preNMDAR debate. 
Highlights (three to five, 85 characters each, with spaces)

- Controversial preNMDARs regulate long and short-term plasticity

- PreNMDARs may signal ionotropically, depending on $\mathrm{Ca}^{2+}$ and $\mathrm{Mg}^{2+}$

- PreNMDARs may signal metabotropically, without $\mathrm{Ca}^{2+}$ or $\mathrm{Mg}^{2+}$ dependence

- Elucidating preNMDAR signaling paths will help settle controversy 


\section{Introduction}

In the classical view, NMDARs act as postsynaptic coincidence detectors that trigger Hebbian plasticity via $\mathrm{Ca}^{2+}$ influx $[1,2]$. PreNMDARs have been relatively overlooked compared to their postsynaptic counterparts, yet have for decades been found at numerous synapse types, where they influence long- as well as short-term plasticity [3-5].

PreNMDARs have been the subject of considerable debate [3-5]. One reason for this is a lack of sufficiently specific experiments, which has raised several questions about preNMDARs: Where precisely at the synapse are they located, and how do they influence synaptic plasticity and transmitter release? Here, we discuss recent studies that address these questions.

\section{PreNMDARs influence long-term plasticity}

Like postsynaptic NMDARs [1,2], preNMDARs determine long-term synaptic plasticity $[3,5,6]$. However, typically only a subset of synaptic boutons express preNMDARs [7], and expression is determined by the postsynaptic target neuron's identity [6,8,9]. For example, synapses from neocortical layer 5 (L5) pyramidal cells (PyCs) to neighboring L5 PyCs express functional preNMDARs, but synapses from PyCs to neighboring basket cells do not [9]. Since preNMDARs are a key determinant of neocortical timing-dependent long-term depression (tLTD) [10], this expression pattern results in synapse-type-specific plasticity $[4,11]$. This heterogeneous expression may also explain why axonal preNMDAR $\mathrm{Ca}^{2+}$ signals have not always been found $[12-15]$.

Another possible explanation for why preNMDARs have been difficult to detect experimentally is that preNMDARs may not always conduct $\mathrm{Ca}^{2+}$ like their postsynaptic counterparts. At connections between cortical layer 4 (L4) spiny stellate cells and layer 2/3 (L2/3) PyCs in somatosensory cortex (Fig. 1) and between CA3 and CA1 neurons in the hippocampus, 
preNMDARs involved in tLTD contain the GluN2C/GluN2D subunits [16,17], which lower $\mathrm{Mg}^{2+}$ sensitivity and $\mathrm{Ca}^{2+}$ permeability [18]. Low preNMDAR $\mathrm{Ca}^{2+}$ conductance has also been suggested at visual cortex L4-L2/3 synapses $[8,19]$, where tLTD depends on both GluN3A- and GluN2B-containing NMDARs, which confer low and high sensitivity to $\mathrm{Mg}^{2+}$, respectively [18]. This form of tLTD may thus involve heterotrimeric preNMDARs that are less sensitive to $\mathrm{Mg}^{2+}$ and that flux less $\mathrm{Ca}^{2+}[18]$.

However, in disagreement with the prior literature [8,19-25], Carter and Jahr [12] recently proposed that preNMDARs are not required for tLTD at L4-L2/3 connections in somatosensory cortex. This finding resembles previously described results at L2/3-L2/3 synapses where, in contrast to L4-L2/3 connections, it is well established that ILTD relies on post- but not presynaptic NMDARs [16,21,26,27]. As Carter and Jahr [12] did not show that the Channelrhodopsin-2 expression was specific to L4, this study may lack specificity for presynaptic cell type, possibly explaining why their findings resemble the previously reported postsynaptic tLTD at L2/3-L2/3 synapses and not preNMDAR-mediated tLTD at L4-L2/3 connections [8,19-25]. Interestingly, Carter and Jahr [12] reported that the postsynaptic NMDARs involved in tLTD at this synapse signaled metabotropically, i.e. without the need for $\mathrm{Ca}^{2+}$ flux (Fig. 1). Although relatively overlooked, metabotropic NMDAR signaling $[28,29]$ — which is mediated by conformational changes rather than ionic flux $[30,31]$ - has interestingly been recently linked to classical hippocampal LTD [32], spine shrinkage [33], and Alzheimer's disease [32,34]. At some synapse, tLTD may thus involve postsynaptic NMDARs signaling metabotropically [12], but it remains unclear precisely which synapses.

Carter and Jahr's [12] study highlights the acute need for an improved understanding of unconventional NMDAR signaling modes [28], because there may be several ways of inducing 
NMDAR-dependent plasticity. For example, although hippocampal CA1 plasticity has traditionally been viewed as postsynaptic [35-37], recent findings by Andrade-Talavera et al. [17] indicate a dissociation: Hippocampal CA1 tLTD depends on preNMDARs and timing-dependent long-term potentiation (tLTP) on postsynaptic NMDARs. A similar dissociation between tLTD and tLTP has previously been found at several neocortical synapses $[10,23,24,38]$, hinting that this is a general principle. Additionally, Andrade-Talavera et al. [17] found that CA3-CA1 tLTD depended on non-postsynaptic ionotropic NMDAR signaling and on presynaptic calcineurin, which could be the target of preNMDAR-mediated $\mathrm{Ca}^{2+}$ (Fig. 1) [17]. Recently, Padamsey et al. [39] demonstrated that GluN1 deletion in CA3 abolished presynaptic LTD, strengthening the evidence for preNMDARs underpinning CA3-CA1 LTD.

PreNMDARs have also been found at several cerebellar synapse types [6]; we focus here on parallel fiber (PF) to Purkinje cell (PuC) synapses. As opposed to neocortical plasticity, PFPuC plasticity follows non-Hebbian rules [40]: Pairing PF and PuC stimulation within a $\pm 100 \mathrm{~ms}$ temporal window elicits LTD. To induce LTP, only PF stimulation is required [41]. Similar to neocortical tLTD, PF-PuC LTD depends on preNMDARs. PF-PuC LTD is expressed postsynaptically via NMDAR-dependent anterograde diffusion of nitric oxide (NO) (Fig. 2) [6,4244]. Recently, using GluN1 deletion in granule cells, Bouvier et al. [43] demonstrated that preNMDARs are essential for PF-PuC LTP. Two-photon $\mathrm{Ca}^{2+}$ imaging showed that only highfrequency activation elicited preNMDAR $\mathrm{Ca}^{2+}$ transients, in keeping with the frequency dependency of LTD and LTP $[43,45]$. PreNMDARs at PFs thus implement a high-pass filter for burst detection, a property presumably inherited from the voltage-dependent $\mathrm{Mg}^{2+}$ blockade of GluN2A-containing NMDARs $[43,45]$. Spread of dendritic NMDAR signals in presynaptic cells $[14,15]$ cannot account for these results, since boutons were severed from somata [as in 46]. 
Using 2-photon imaging, Bouvier et al. [43] only found preNMDARs in a subset of PF boutons. This heterogeneity of preNMDAR expression is reminiscent of that reported in visual cortex $[8,9]$. In the latter case, however, preNMDAR expression is specific for synapse type. Nevertheless, heterogeneity may be a general principle governing preNMDARs [4], which sets them apart from postsynaptic NMDARs $[9,47]$.

\section{PreNMDARs also influence short-term plasticity}

In addition to LTP and LTD, preNMDARs regulate short-term plasticity (STP) [10,47], encompassing both facilitation and depression, with a time course ranging from milliseconds to minutes [48]. STP adds diversity of signaling in local circuits, because synapses from the same neuron onto different target neuron types can express widely different forms of STP [9]. This means STP is synapse type specific [11], just like long-term plasticity [4].

Since preNMDARs are typically sensitive to $\mathrm{Mg}^{2+}[3,5]$, at least two action potentials (APs) in rapid succession should be required to activate preNMDARs, at least if they operate as autoreceptors [although see 49,50]. This is because the AP is so brief that when glutamate binds preNMDARs, they remain $\mathrm{Mg}^{2+}$ blocked unless depolarized by subsequent APs. In agreement, such frequency-dependent activation of preNMDARs has been reported in visual cortex $[8,10,47]$, hippocampus [51] and cerebellum [43,45]. $\mathrm{Mg}^{2+}$-sensitive preNMDARs can thus be thought of as detectors of high-frequency presynaptic firing. In neocortex, the critical frequency for preNMDAR activation is $\sim 8 \mathrm{~Hz}$ [47] (although hippocampal preNMDARs bandpass filter around $\sim 5 \mathrm{~Hz}$ [51]).

One apparent caveat with this interpretation is that preNMDARs apparently modify release probability $\left(p_{r}\right)$ at the first AP in a high-frequency train [8-10,21,47], when preNMDARs should presumably still be $\mathrm{Mg}^{2+}$ blocked. Recently, Abrahamsson et al. [47] proposed a solution to this 
conundrum: PreNMDARs may regulate $p_{r}$ indirectly, by modifying the replenishment rate of the readily releasable pool (RRP) (Fig. 3), which would affect $p_{r}$ at all APs in a high-frequency train, including the first. This interpretation may hold true for preNMDARs with frequency-dependent regulation of STP, such as those at L4-L2/3 synapses $[8,21]$ or at hippocampal CA3-CA1 synapses [51]. As a general principle, preNMDARs may thus serve to upregulate RRP replenishment rates during periods of elevated activity, to sustain $p_{r}$.

\section{Regulation of spontaneous release by preNMDARs}

Neocortical preNMDARs generally modulate both evoked and spontaneous release [8$10,19,47]$. This may seem peculiar, since preNMDAR regulation of evoked release requires presynaptic spiking above $\sim 8 \mathrm{~Hz}$ [47], but spontaneous release rates are typically considerably lower $[9,10]$. Yet, the preNMDARs that regulate evoked and spontaneous release both contain the GluN2B subunit [10], implying that preNMDAR regulation of spontaneous release should also be $\mathrm{Mg}^{2+}$ sensitive [18] [but see 19]. This apparent discrepancy has been a long-standing issue in the field $[3,5,10,21]$.

Although evoked and spontaneous release were initially thought to rely on the same molecular machinery, the emerging consensus is that the two forms of release employ distinct pathways [52]. In agreement, Abrahamsson et al. [47] found that preNMDARs at excitatory synapses onto visual cortex L5 PyCs regulate evoked and spontaneous release via non-overlapping pathways (Fig. 3). PreNMDARs rely on the active-zone protein RIM1 $\alpha \beta$ [53] to regulate evoked but not spontaneous release. Conversely, preNMDARs require JNK2 [54] to control spontaneous release — as previously shown in entorhinal cortex [55] — but not evoked release. These findings thus revealed a double dissociation of preNMDAR signaling in controlling transmitter release. 
Still, the absence of frequency dependence in preNMDAR regulation of spontaneous release was enigmatic. An explanation might be found in metabotropic NMDAR signaling, since it is not $\mathrm{Mg}^{2+}$ dependent [32]. Consistent with metabotropic signaling, Abrahamsson et al. [47] found that spontaneous release in L5 PyCs was not sensitive to $\mathrm{Mg}^{2+}$ wash-out or MK-801 washin. This was furthermore in agreement with earlier findings that preNMDAR control of spontaneous release does not rely on $\mathrm{Ca}^{2+}$ [56]. Taken together, these findings suggest that preNMDARs depend on RIM1 $\alpha \beta$ and relief of $\mathrm{Mg}^{2+}$ blockade to control evoked release, but on JNK2 and metabotropic signaling to regulate spontaneous release [47] (Fig. 3). This interpretation helps to explain the long-standing issue of how preNMDAR-dependent regulation of evoked by not spontaneous release requires a critical frequency $[3,5,10,21]$.

It is not clear, however, that the preNMDARs controlling evoked and spontaneous release are the same. In hippocampal cultures, for example, spontaneous and evoked release sites do not necessarily overlap [57]. A simple interpretation is that the same population of preNMDARs regulates both spontaneous and evoked release via two different paths (Fig. 3). Another possibility is that two preNMDAR populations are physically separated, e.g. by RIM nanocolumns [58]. A third possibility is that NMDARs modulate different bouton populations, one responsible for evoked release and the other for spontaneous release [57,59]. In any of these scenarios, the preNMDARs modulating evoked release would presumably be GluN1-GluN2B heterodimers, but the preNMDARs modulating spontaneous release could also be GluN1-N2B/N2C/N2D or GluN3containing heterotrimers. These heterotrimers would explain the GluN2B pharmacological profile in combination with low $\mathrm{Mg}^{2+}$ sensitivity [18]. Finally, another possibility is that these vesicle pools are the same, but that the $\mathrm{Ca}^{2+}$ sensor for evoked and spontaneous release are distinct [60]. 
The schematic in Fig. $\mathbf{3}$ is thus merely a parsimonious working model to illustrate the findings of Abrahamsson et al. [47].

\section{Conclusions and future directions}

For a long time, the preNMDAR field suffered from a lack of cell-type specific tools and powerful imaging techniques. However, it is currently well established that preNMDARs play key roles in different brain regions during both long-term and short-term plasticity [3-6]. Until recently, one outstanding question was how preNMDARs can modulate both evoked and spontaneous release when the former but not the latter is frequency dependent [3,5,10,21]. Abrahamsson et al. [47] addressed this question and found that preNMDARs employ specific and distinct pathways regulating evoked and spontaneous release separately.

This discovery, however, raises several other issues. Are these preNMDARs involved in evoked and spontaneous release the same? Do classical properties of postsynaptic NMDARs apply to preNMDARs? For example, preNMDARs have mixed pharmacological profiles, with reduced $\mathrm{Ca}^{2+}$ conductance, which may make them distinct from postsynaptic NMDARs $[8,16,17,19]$. PreNMDARs can apparently also signal metabotropically or ionotropically to control different aspects of synaptic release [47].

It is tempting to compare the preNMDAR debate with the controversy surrounding the preversus postsynaptic location of long-term synaptic plasticity, which was once quite heated [3537]. Over the past two decades, however, this controversy has gradually given way to the realization that plasticity is determined by many different factors, such as animal age, induction protocol, and synapse type [35-37]. The controversy surrounding preNMDARs has echoed this debate. We believe the preNMDAR debate is going to be resolved in a similar manner: with 
preNMDARs, like with LTP and LTD [61] , there is an embarrassment of riches. This diversity has ensured long-standing disagreement among neuroscientists. For this reason, we argue that elucidating unconventional NMDAR signaling — presynaptically as well as metabotropically — will help resolve the preNMDAR debate [28].

\section{Acknowledgements}

We thank Alanna Watt, Jennifer Brock, and Christina Chou for help and discussions. This work was funded by the NIH EY025668 (G.B.), the Allen Institute for Brain Science (R.S.L.), MINECO BFU2015-68655-P (A.R.-M.), a Royal Society International Exchanges Grant (A.R.M. and O.P.), the BBSRC (O.P.), CIHR OG 126137 (P.J.S.), CIHR NIA 288936 (P.J.S.), NSERC DG 2017-04730 (P.J.S.), and NSERC DAS 2017-507818 (P.J.S.). The authors declare no conflicts of interest. 
Figure legends

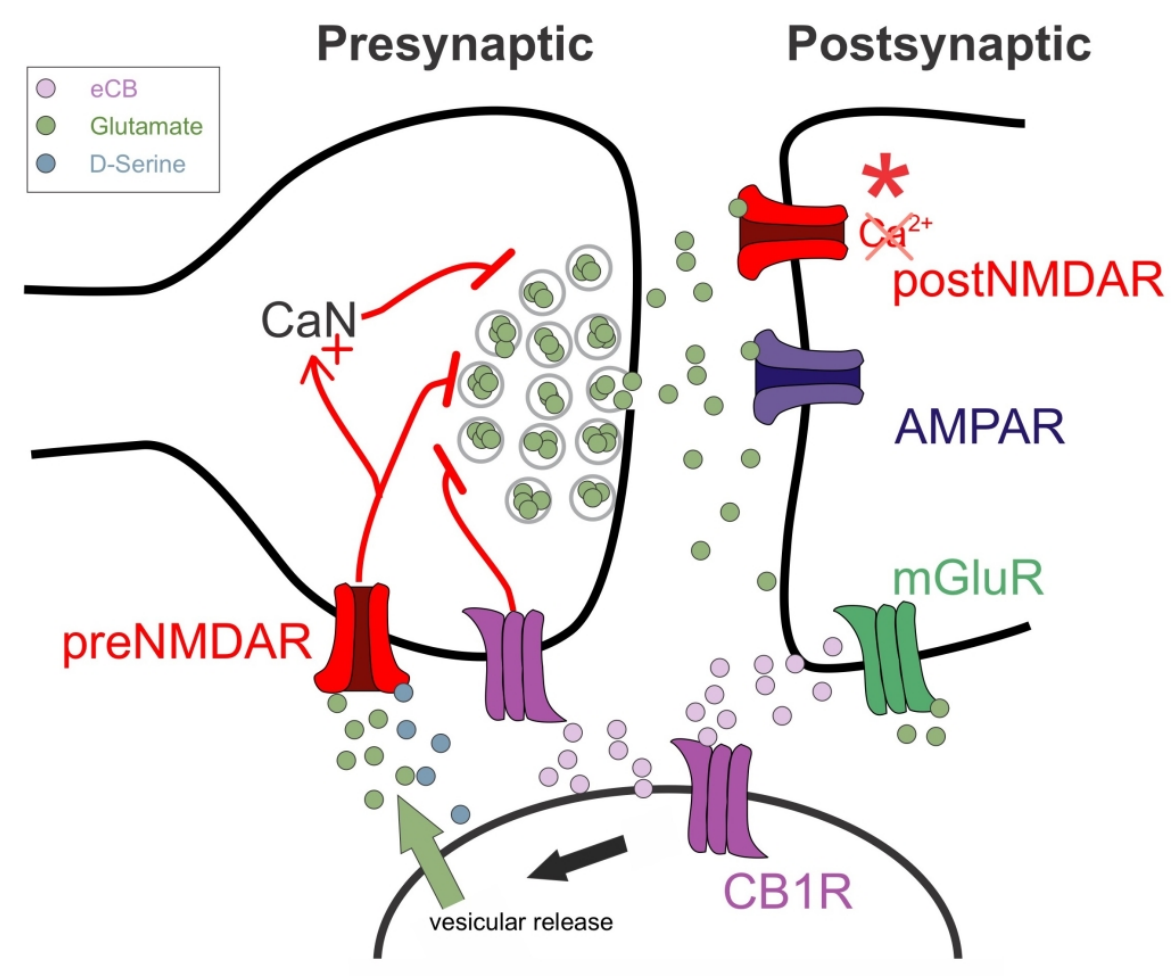

Astrocyte

Figure 1 - Differing models of $L L T D$ at $L 4$ to $L 2 / 3$ connections

In tLTD induction, presynaptic NMDARs and cannabinoid receptors (CB1Rs) are typically required for tLTD [although see 22]. CB1Rs are expressed both on L4 boutons and astrocytic processes, where they modulate the presynaptic release or elicit release of glutamate and D-serine, respectively. PreNMDAR-mediated $\mathrm{Ca}^{2+}$ activates calcineurin $(\mathrm{CaN})$, which triggers a reduction in $p_{r}$. This model is based on many studies over two decades $[8,10,19-25]$. In contrast to these studies, Carter and Jahr [12] proposed that postsynaptic NMDARs (postNMDAR) but not preNMDARs trigger tLTD via metabotropic signaling (asterisk). They did not explore the expression mechanism [12]. 


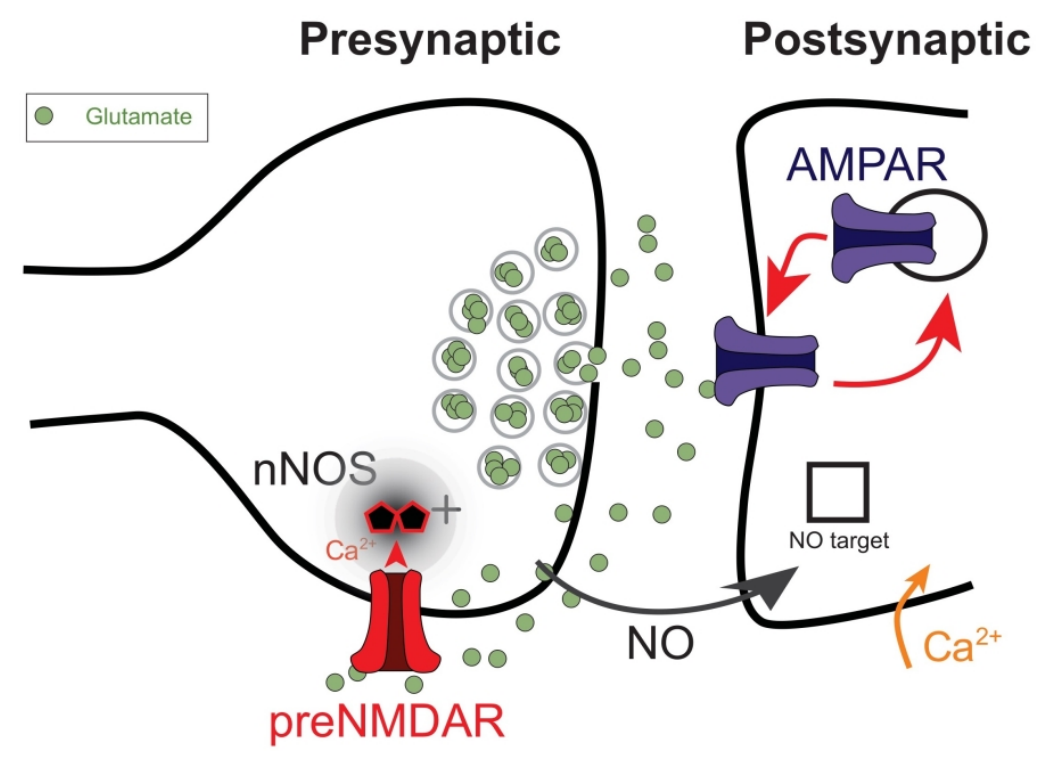

Figure 2 - Cerebellar long-term plasticity relies on preNMDARs and transsynaptic NO signaling

At PF-PuCs synapses, preNMDARs implement a bidirectional high-pass filter synaptic plasticity rule that is expressed postsynaptically $[43,45]$. High-frequency PF bursts activate preNMDARs, resulting in $\mathrm{Ca}^{2+}$ influx that activates neuronal nitric oxide synthase (nNOS), which is typically tightly coupled to NMDARs [62]. Nitric oxide (NO, black shadow) diffuses anterogradely to the postsynaptic side (black arrow). PF bursting produces NO in an NMDARdependent manner [44], while NO-mediated activation of guanylate cyclase is necessary for LTD induction $[63,64]$. In contrast, the targets of NO in LTP induction are unknown (black square: NO target), although guanylate cyclase is not required [42]. 


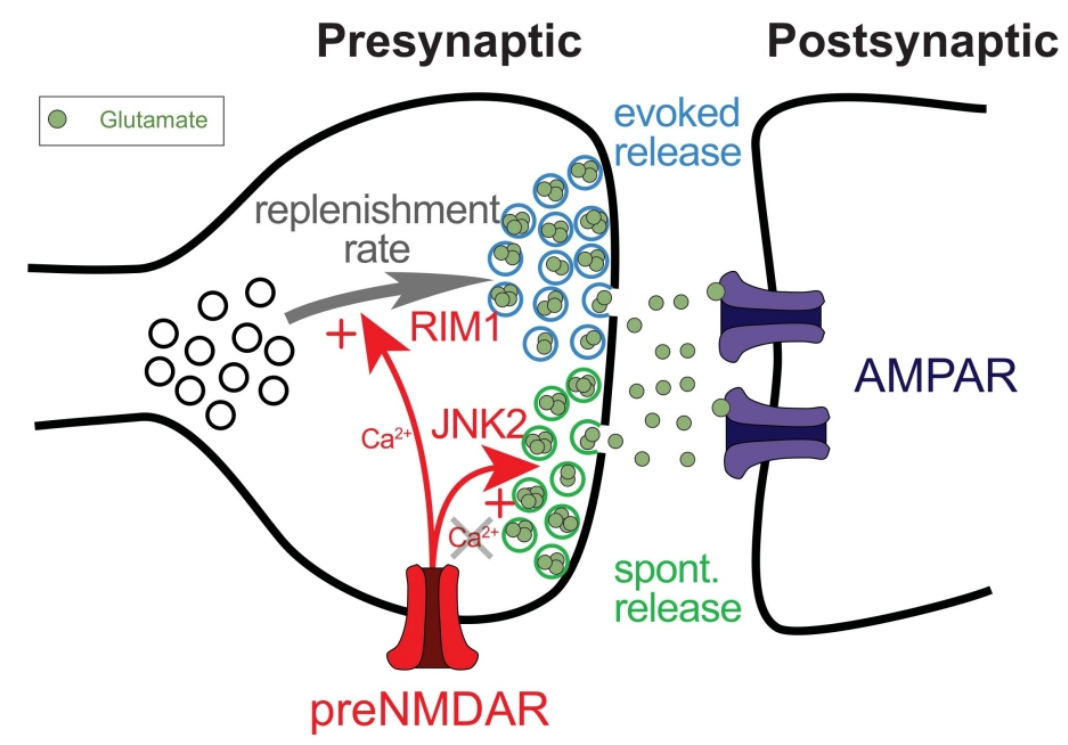

Figure 3 - At L5 excitatory connections, preNMDARs differentially control evoked and spontaneous release

Neocortical L5 PyC PreNMDARs rely on RIM1 $\alpha \beta$ and $\mathrm{Ca}^{2+}$ to upregulate the RRP replenishment rate, which sustains evoked release during periods of high-frequency firing. The upregulation of spontaneous release is separately controlled via JNK2 without $\mathrm{Ca}^{2+}$ or $\mathrm{Mg}^{2+}$ dependence $[47,56]$. This working model explains the different frequency dependencies of preNMDAR control of spontaneous and evoked release, and furthermore supports the view that spontaneous and evoked release are distinct processes [52]. 


\section{Recommended reading articles}

- of special interest

• of outstanding interest

•• Abrahamsson et al., 2017 [47]

The authors find that neocortical preNMDAR regulation of evoked and spontaneous release is doubly dissociated: preNMDAR regulation of evoked relies on RIM1 $\alpha \beta$, whereas that of spontaneous depends on JNK2. To sustain rapid evoked release, preNMDARs boost $p_{r}$ indirectly by upregulating RRP replenishment rate. Finally, spontaneous release regulation was not $\mathrm{Mg}^{2+}$ sensitive, suggesting metabotropic signaling.

•• Andrade-Talavera et al., 2016 [17]

The authors investigated the requirements for induction of tLTP and tLTD at CA3-CA1 synapses in P12-18 mouse hippocampus. They found that tLTP requires postsynaptic NMDARs containing GluN2A and GluN2B subunits, while tLTD depends on non-postsynaptic NMDARs containing GluN2C or GluN2D subunits.

- Aow et al., 2015 [31]

Using FRET between fluorescently tagged GluN1 subunit cytoplasmic domains and Protein Phosphatase 1 (PP1), Aow et al. obtain findings in hippocampal cultures consistent with a transient movement of PP1 by NMDAR ligand binding in the absence of $\mathrm{Ca}^{2+}$ flux. As PP1 drives AMPA receptor removal, this suggests metabotropic NMDAR signaling in LTD [also see 30].

•• Bouvier et al., 2016 [43]

Using 2-photon $\mathrm{Ca}^{2+}$ imaging, cell-type specific deletion of preNMDARs, and computer modelling, the authors show that cerebellar PF-PuC preNMDARs underlie LTP. They show direct 
evidence for $\mathrm{Ca}^{2+}$ entry via preNMDARs in a subpopulation of $\mathrm{PF}$ varicosities during highfrequency bursts.

- Carter and Jahr, 2016 [12]

Using 2-photon $\mathrm{Ca}^{2+}$ imaging, optogenetics, and slice electrophysiology at barrel cortex L4-L2/3 synapses, the authors found evidence that post- rather than presynaptic NMDARs underlie tLTD induction. They propose that postsynaptic NMDARs signal metabotropically, independent of $\mathrm{Ca}^{2+}$.

- Dore et al., 2015 [30]

Using FRET between fluorescently tagged GluN1 subunit cytoplasmic domains, Dore and colleagues show in hippocampal culture that glutamate binding can transmit conformational information into the cell in the absence of ion flow, consistent with metabotropic NMDAR signaling [also see 31].

• Nistico et al., 2015 [55]

Using biochemical, morphological, and functional approaches, Nisticò et al. find in entorhinal cortex that JNK and its scaffold protein JIP1 are expressed presynaptically. They also show that putative preNMDARs rely on JNK2 to modulate NMDA-evoked as well as spontaneous glutamate release, thus identifying a key preNMDAR signaling path.

- Padamsey et al., 2017 [39]

The authors found that presynaptic CA3-CA1 LTD relied on preNMDARs activated by glutamate release. In contrast, presynaptic CA3-CA1 LTP did not require glutamate signaling. Presynaptic LTP depended on activation of postsynaptic L-type $\mathrm{Ca}^{2+}$ channels that trigger retrograde nitric-oxide signaling. 
- Stein et al., 2015 [33]

Spine elimination is widely thought to depend on NMDAR-mediated $\mathrm{Ca}^{2+}$ influx in conjunction with LTD. Combining 2-photon uncaging and time-lapse imaging with slice electrophysiology, Stein et al. suggest that in rat hippocampus, metabotropic NMDAR signaling drive spine shrinkage and LTD independent of $\mathrm{Ca}^{2+}$.

\section{References}

1. Maheux J, Froemke RC, Sjöström PJ: Functional plasticity at dendritic synapses. In Dendrites, edn 3. Edited by Stuart G, Spruston N, Häusser M: Oxford University Press; 2016:465-498.

2. Sjöström PJ, Rancz EA, Roth A, Häusser M: Dendritic Excitability and Synaptic Plasticity. Physiological Reviews 2008, 88:769-840.

3. Banerjee A, Larsen RS, Philpot BD, Paulsen O: Roles of Presynaptic NMDA Receptors in Neurotransmission and Plasticity. Trends Neurosci 2016, 39:26-39.

4. Larsen RS, Sjöström PJ: Synapse-type-specific plasticity in local circuits. Curr Opin Neurobiol 2015, 35:127-135.

5. Duguid I, Sjöström PJ: Novel presynaptic mechanisms for coincidence detection in synaptic plasticity. Curr Opin Neurobiol 2006, 16:312-322.

6. Bouvier G, Bidoret C, Casado M, Paoletti P: Presynaptic NMDA receptors: Roles and rules. Neuroscience 2015, 311:322-340.

7. Rossi B, Ogden D, Llano I, Tan YP, Marty A, Collin T: Current and calcium responses to local activation of axonal NMDA receptors in developing cerebellar molecular layer interneurons. PLoS One 2012, 7:e39983.

8. Larsen RS, Smith IT, Miriyala J, Han JE, Corlew RJ, Smith SL, Philpot BD: Synapse-specific control of experience-dependent plasticity by presynaptic NMDA receptors. Neuron 2014, 83:879-893.

9. Buchanan KA, Blackman AV, Moreau AW, Elgar D, Costa RP, Lalanne T, Tudor Jones AA, Oyrer J, Sjöström PJ: Target-Specific Expression of Presynaptic NMDA Receptors in Neocortical Microcircuits. Neuron 2012, 75:451-466.

10. Sjöström PJ, Turrigiano GG, Nelson SB: Neocortical LTD via coincident activation of presynaptic NMDA and cannabinoid receptors. Neuron 2003, 39:641-654.

11. Blackman AV, Abrahamsson T, Costa RP, Lalanne T, Sjöström PJ: Target Cell-Specific Short-Term Plasticity in Local Circuits. Frontiers in Synaptic Neuroscience 2013, 5:113.

12. Carter BC, Jahr CE: Postsynaptic, not presynaptic NMDA receptors are required for spike-timing-dependent LTD induction. Nat Neurosci 2016, 19:1218-1224.

13. Christie JM, Jahr CE: Selective expression of ligand-gated ion channels in L5 pyramidal cell axons. $J$ Neurosci 2009, 29:11441-11450.

14. Christie JM, Jahr CE: Dendritic NMDA receptors activate axonal calcium channels. Neuron 2008, 60:298-307. 
15. Christie JM, Chiu DN, Jahr CE: $\mathbf{C a}^{2+}$-dependent enhancement of release by subthreshold somatic depolarization. Nat Neurosci 2011, 14:62-68.

16. Banerjee A, Meredith RM, Rodríguez-Moreno A, Mierau SB, Auberson YP, Paulsen O: Double Dissociation of Spike Timing-Dependent Potentiation and Depression by Subunit-Preferring NMDA Receptor Antagonists in Mouse Barrel Cortex. Cereb Cortex 2009.

17. Andrade-Talavera Y, Duque-Feria P, Paulsen O, Rodríguez-Moreno A: Presynaptic Spike Timing-Dependent Long-Term Depression in the Mouse Hippocampus. Cereb Cortex 2016, 26:3637-3654.

18. Paoletti $\mathrm{P}$, Bellone $\mathrm{C}$, Zhou Q: NMDA receptor subunit diversity: impact on receptor properties, synaptic plasticity and disease. Nat Rev Neurosci 2013, 14:383-400.

19. Larsen RS, Corlew RJ, Henson MA, Roberts AC, Mishina M, Watanabe M, Lipton SA, Nakanishi N, Perez-Otano I, Weinberg RJ, et al.: NR3A-containing NMDARs promote neurotransmitter release and spike timing-dependent plasticity. Nat Neurosci 2011, 14:338-344.

20. Bender VA, Bender KJ, Brasier DJ, Feldman DE: Two coincidence detectors for spike timing-dependent plasticity in somatosensory cortex. $J$ Neurosci 2006, 26:4166-4177.

21. Brasier DJ, Feldman DE: Synapse-specific expression of functional presynaptic NMDA receptors in rat somatosensory cortex. J Neurosci 2008, 28:2199-2211.

22. Rodríguez-Moreno A, Gonzalez-Rueda A, Banerjee A, Upton AL, Craig MT, Paulsen O: Presynaptic self-depression at developing neocortical synapses. Neuron 2013, 77:3542.

23. Rodríguez-Moreno A, Kohl MM, Reeve JE, Eaton TR, Collins HA, Anderson HL, Paulsen O: Presynaptic induction and expression of timing-dependent long-term depression demonstrated by compartment-specific photorelease of a use-dependent NMDA receptor antagonist. $J$ Neurosci 2011, 31:8564-8569.

24. Rodríguez-Moreno A, Paulsen O: Spike timing-dependent long-term depression requires presynaptic NMDA receptors. Nat Neurosci 2008, 11:744-745.

25. Min R, Nevian T: Astrocyte signaling controls spike timing-dependent depression at neocortical synapses. Nat Neurosci 2012, 15:746-753.

26. Froemke RC, Poo MM, Dan Y: Spike-timing-dependent synaptic plasticity depends on dendritic location. Nature 2005, 434:221-225.

27. Banerjee A, Gonzalez-Rueda A, Sampaio-Baptista C, Paulsen O, Rodriguez-Moreno A: Distinct mechanisms of spike timing-dependent $L T D$ at vertical and horizontal inputs onto L2/3 pyramidal neurons in mouse barrel cortex. Physiol Rep 2014, 2:e00271.

28. Dore K, Stein IS, Brock JA, Castillo PE, Zito K, Sjöström PJ: Unconventional NMDA Receptor Signaling. J Neurosci 2017, 37:10800-10807.

29. Dore K, Aow J, Malinow R: The Emergence of NMDA Receptor Metabotropic Function: Insights from Imaging. Front Synaptic Neurosci 2016, 8:20.

30. Dore K, Aow J, Malinow R: Agonist binding to the NMDA receptor drives movement of its cytoplasmic domain without ion flow. Proc Natl Acad Sci U S A 2015, 112:1470514710.

31. Aow J, Dore K, Malinow R: Conformational signaling required for synaptic plasticity by the NMDA receptor complex. Proc Natl Acad Sci U S A 2015, 112:14711-14716. 
32. Nabavi S, Kessels HW, Alfonso S, Aow J, Fox R, Malinow R: Metabotropic NMDA receptor function is required for NMDA receptor-dependent long-term depression. Proc Natl Acad Sci U S A 2013, 110:4027-4032.

33. Stein IS, Gray JA, Zito K: Non-Ionotropic NMDA Receptor Signaling Drives ActivityInduced Dendritic Spine Shrinkage. J Neurosci 2015, 35:12303-12308.

34. Kessels HW, Nabavi S, Malinow R: Metabotropic NMDA receptor function is required for beta-amyloid-induced synaptic depression. Proc Natl Acad Sci U S A 2013, 110:40334038.

35. Kullmann DM: The Mother of All Battles 20 years on: is LTP expressed pre- or postsynaptically? J Physiol 2012, 590:2213-2216.

36. Padamsey $Z$, Emptage $N$ : Two sides to long-term potentiation: a view towards reconciliation. Philos Trans R Soc Lond B Biol Sci 2014, 369:20130154.

37. MacDougall MJ, Fine A: The expression of long-term potentiation: reconciling the preists and the postivists. Philos Trans R Soc Lond B Biol Sci 2014, 369:20130135.

38. Sjöström PJ, Turrigiano GG, Nelson SB: Multiple forms of long-term plasticity at unitary neocortical layer 5 synapses. Neuropharmacology 2007, 52:176-184.

39. Padamsey Z, Tong R, Emptage N: Glutamate is required for depression but not potentiation of long-term presynaptic function. Elife 2017,6 .

40. Jörntell H, Hansel C: Synaptic memories upside down: bidirectional plasticity at cerebellar parallel fiber-Purkinje cell synapses. Neuron 2006, 52:227-238.

41. Safo P, Regehr WG: Timing dependence of the induction of cerebellar LTD. Neuropharmacology 2008, 54:213-218.

42. Lev-Ram V, Wong ST, Storm DR, Tsien RY: A new form of cerebellar long-term potentiation is postsynaptic and depends on nitric oxide but not cAMP. Proc Natl Acad Sci U S A 2002, 99:8389-8393.

43. Bouvier G, Higgins D, Spolidoro M, Carrel D, Mathieu B, Lena C, Dieudonne S, Barbour B, Brunel N, Casado M: Burst-Dependent Bidirectional Plasticity in the Cerebellum Is Driven by Presynaptic NMDA Receptors. Cell Rep 2016, 15:104-116.

44. Wang DJ, Su LD, Wang YN, Yang D, Sun CL, Zhou L, Wang XX, Shen Y: Long-term potentiation at cerebellar parallel fiber-Purkinje cell synapses requires presynaptic and postsynaptic signaling cascades. $J$ Neurosci 2014 , 34:2355-2364.

45. Bidoret C, Ayon A, Barbour B, Casado M: Presynaptic NR2A-containing NMDA receptors implement a high-pass filter synaptic plasticity rule. Proc Natl Acad Sci U S A 2009, 106:14126-14131.

46. Park H, Popescu A, Poo MM: Essential Role of Presynaptic NMDA Receptors in ActivityDependent BDNF Secretion and Corticostriatal LTP. Neuron 2014, 84:1009-1022.

47. Abrahamsson T, Chou CYC, Li SY, Mancino A, Costa RP, Brock JA, Nuro E, Buchanan KA, Elgar D, Blackman AV, et al.: Differential Regulation of Evoked and Spontaneous Release by Presynaptic NMDA Receptors. Neuron 2017, 96:839-855 e835.

48. Abbott LF, Regehr WG: Synaptic computation. Nature 2004, 431:796-803.

49. Humeau Y, Shaban H, Bissière S, Lüthi A: Presynaptic induction of heterosynaptic associative plasticity in the mammalian brain. Nature 2003, 426:841-845.

50. Duguid IC, Smart TG: Retrograde activation of presynaptic NMDA receptors enhances GABA release at cerebellar interneuron-Purkinje cell synapses. Nat.Neurosci. 2004, 7:525-533. 
51. McGuinness L, Taylor C, Taylor RD, Yau C, Langenhan T, Hart ML, Christian H, Tynan PW, Donnelly $\mathrm{P}$, Emptage NJ: Presynaptic NMDARs in the hippocampus facilitate transmitter release at theta frequency. Neuron 2010, 68:1109-1127.

52. Kavalali ET: The mechanisms and functions of spontaneous neurotransmitter release. Nat Rev Neurosci 2015, 16:5-16.

53. Südhof TC: The presynaptic active zone. Neuron 2012, 75:11-25.

54. Yamasaki T, Kawasaki H, Nishina H: Diverse Roles of JNK and MKK Pathways in the Brain. J Signal Transduct 2012, 2012:459265.

55. Nisticò R, Florenzano F, Mango D, Ferraina C, Grilli M, Di Prisco S, Nobili A, Saccucci S, D'Amelio M, Morbin M, et al.: Presynaptic c-Jun N-terminal Kinase 2 regulates NMDA receptor-dependent glutamate release. Sci Rep 2015, 5:9035.

56. Kunz PA, Roberts AC, Philpot BD: Presynaptic NMDA receptor mechanisms for enhancing spontaneous neurotransmitter release. $J$ Neurosci 2013, 33:7762-7769.

57. Reese AL, Kavalali ET: Single synapse evaluation of the postsynaptic NMDA receptors targeted by evoked and spontaneous neurotransmission. eLife 2016, 5.

58. Tang AH, Chen H, Li TP, Metzbower SR, MacGillavry HD, Blanpied TA: A trans-synaptic nanocolumn aligns neurotransmitter release to receptors. Nature 2016, 536:210-214.

59. Melom JE, Akbergenova Y, Gavornik JP, Littleton JT: Spontaneous and evoked release are independently regulated at individual active zones. $J$ Neurosci 2013 , 33:17253-17263.

60. Groffen AJ, Martens S, Diez Arazola R, Cornelisse LN, Lozovaya N, de Jong AP, Goriounova NA, Habets RL, Takai Y, Borst JG, et al.: Doc2b is a high-affinity $\mathbf{C a}^{2+}$ sensor for spontaneous neurotransmitter release. Science 2010, 327:1614-1618.

61. Malenka RC, Bear MF: LTP and LTD: an embarrassment of riches. Neuron 2004, 44:5-21.

62. Christopherson KS, Hillier BJ, Lim WA, Bredt DS: PSD-95 assembles a ternary complex with the N-methyl-D-aspartic acid receptor and a bivalent neuronal NO synthase PDZ domain. J Biol Chem 1999, 274:27467-27473.

63. Boxall AR, Garthwaite J: Long-term depression in rat cerebellum requires both NO synthase and NO-sensitive guanylyl cyclase. Eur J Neurosci 1996, 8:2209-2212.

64. Lev-Ram V, Jiang T, Wood J, Lawrence DS, Tsien RY: Synergies and coincidence requirements between NO, cGMP, and $\mathrm{Ca}^{2+}$ in the induction of cerebellar long-term depression. Neuron 1997, 18:1025-1038. 